\title{
Fusion hindrance in light and heavy systems
}

\author{
Giovanna Montagnoli ${ }^{1,2, *}$ \\ ${ }^{1}$ University of Padova \\ ${ }^{2}$ INFN Sezione di Padova
}

\begin{abstract}
.
The phenomenon of hindrance in sub-barrier heavy-ion fusion has been confirmed by several experimental evidences and it is now recognised as a general phenomenon of heavy-ion fusion process. In many cases the signature of fusion hindrance lies in the trend of the logarithmic slope of the excitation function and of the $S$ factor at low energies. The comparison with standard Coupled-Channels calculations is a more quantitative evidence for its existence. In many medium-heavy systems the hindrance effect has been recognised with different features depending on the various couplings to the inelastic and transfer channels. Different theoretical approaches have been proposed to explain the hindrance but the underlying physics is still a matter of debate. Hindrance is observed in light systems, independent of the sign of the fusion $Q$-value, with different features. In the case of the ${ }^{12} \mathrm{C}+{ }^{30} \mathrm{Si}$ system the effect is small but it is clearly observed. Near-by cases show evidence for systematic behaviours. A very recent experiment has concerned the lighter case ${ }^{12} \mathrm{C}+{ }^{24} \mathrm{Mg}$ where hindrance shows up clearly, because a maximum of the $S$ factor appears already at a relatively high cross section $\sigma=1.6$ $\mathrm{mb}$. The consequences for the dynamics of stellar evolution have to be clarified by further experimental and theoretical work.
\end{abstract}

\section{Introduction}

Heavy-ion fusion reactions near and below the barrier are typical phenomena where the interplay of reaction dynamics and nuclear structure leads to a large enhancement of the fusion probability with respect to the one-dimensional quantum tunnelling limit. An important step forward in the fusion studies has been the introduction of the concept of barrier distribution [1] generated by the channel couplings and the possibility to obtain it from the experimental excitation functions. The barrier distribution has been exploited to disentangle the influence of the various coupled channels and its sensitivity to the static nuclear deformation [2], to the collective multi-phonon excitations [3] and to the transfer channels with positive $Q$-value [4], is remarkable.

Extending down in energy it has been observed that the fusion excitation functions of many systems decrease more steeply than the predictions of standard Coupled-Channels (CC) calculations based on a Wood-Saxon potential. This phenomenon known as fusion hindrance has been recognised as a general feature forcing us to rethink the shape of the inner part of the potential barrier as some theoretical models indicate.

In this talk the cases of ${ }^{12} \mathrm{C}+{ }^{30} \mathrm{Si},{ }^{24} \mathrm{Mg}$, recently studied at LNL, will be presented and discussed, showing the sub-barrier trend of their excitation function and of the $S$ factor. Indeed, if the hindrance phenomenon exists in light systems, the fusion cross sections (viz. the $S$ factors)

\footnotetext{
*e-mail: montagnoli@pd.infn.it
}

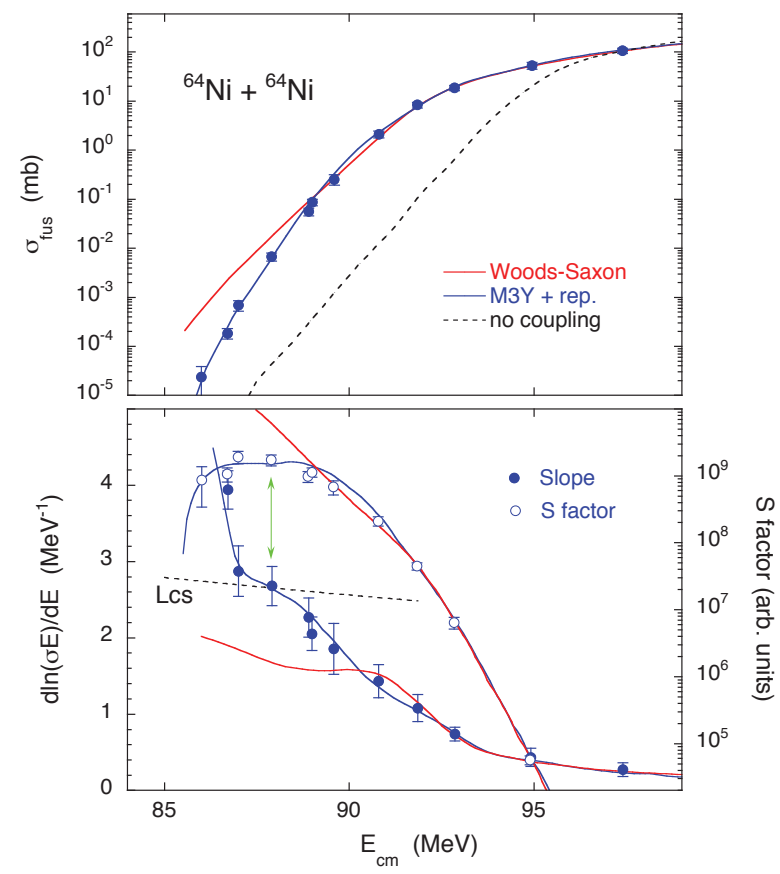

Figure 1. Fusion excitation function (upper panel), logarithmic slope and astrophysical $S$ factor (lower panel) of ${ }^{64} \mathrm{Ni}+$ ${ }^{64} \mathrm{Ni}[10]$.

near the Gamow peak might be substantially smaller than expected previously by simple extrapolations of the highenergy trends, where sets of measurements already exist. Fusion reactions between light heavy ions have a prominent role in the dynamics of stellar evolution. 
It was pointed out [5] that reactions such as ${ }^{12} \mathrm{C}+{ }^{12} \mathrm{C}$, ${ }^{12} \mathrm{C}+{ }^{16} \mathrm{O}$ and ${ }^{16} \mathrm{O}+{ }^{16} \mathrm{O}$ (all of them have positive $\mathrm{Q}$ values for fusion) are important for the evolution of massive stars, beyond the helium burning, and the associated nucleosynthesis [6]. The existence of hindrance in these cases would lead to significant changes of the abundances of many isotopes. The two medium-light systems ${ }^{12} \mathrm{C}+$ ${ }^{30} \mathrm{Si},{ }^{24} \mathrm{Mg}$ may be helpful to establish a reliable systematic behaviour to guide the extrapolation to lighter cases of astrophysical interest.

\section{The phenomenon of fusion hindrance at deep sub-barrier energies}

Hindrance of heavy-ion fusion at extreme sub-barrier energies, characterized by a steep fall off of the fusion cross section with decreasing energy, was discovered 15 years ago [6]. By plotting the cross section in terms of the $S$ factor, $S(E)=\sigma E \exp (2 \pi \eta)$, where $\eta$ is the Sommerfeld parameter and $E$ is the center of mass energy, fusion hindrance is easily recognized by a maximum of $S(E)$ at an energy $E_{s}[7-9]$.
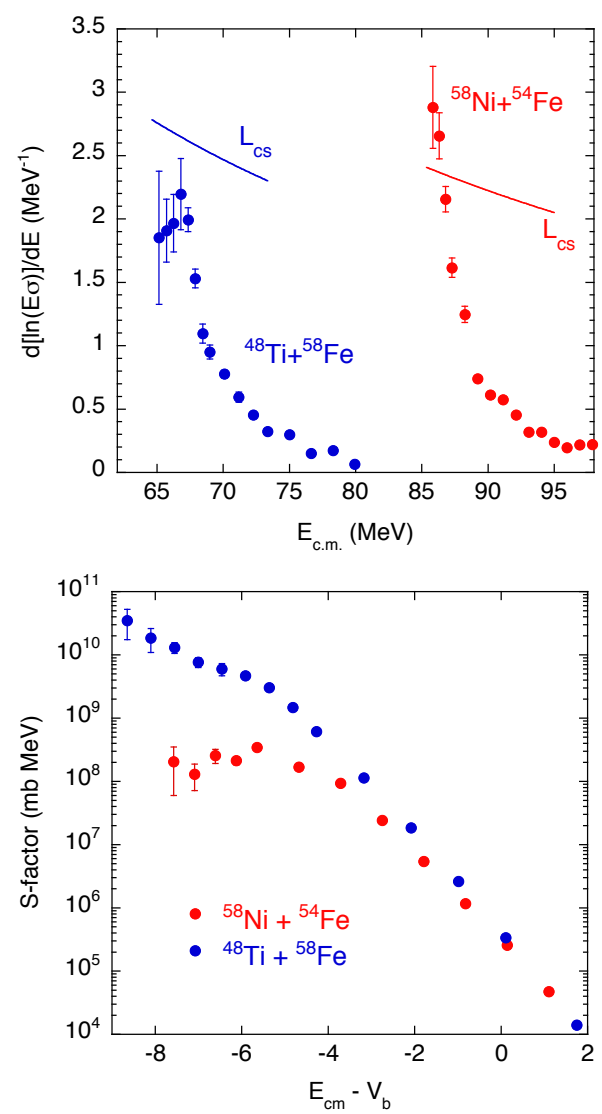

Figure 2. Logarithmic slopes (upper panel) and the $S$ factor (lower panel) of ${ }^{48} \mathrm{Ti}+{ }^{58} \mathrm{Fe}$ and ${ }^{58} \mathrm{Ni}+{ }^{54} \mathrm{Fe}[11,12]$.

This phenomenon was first studied in medium-heavy systems. In this mass region, the fusion $Q$ value is always negative, so that the $S$ factor is 0 , when the incident energy approaches $E=-Q$. Thus, under such conditions an $S$ factor maximum is unavoidable [7-9]. The classical example is ${ }^{64} \mathrm{Ni}+{ }^{64} \mathrm{Ni}$ where the fusion cross section was measured down to about $20 \mathrm{nb}$. This is shown in Fig. 1. The excitation function decreases more steeply than the prediction of a standard CC calculation. This is better evidenced by the representation in terms of logarithmic derivative $L(E)$ of the excitation function which is reported in the lower panel of Fig. 1. It is known [7] that when the slope reaches the value $L_{C S}=\pi \eta / E$ the $S$ factor develops a maximum and the corresponding energy is usually taken as the threshold for the hindrance effect.

Even for the hindrance phenomenon the role of nuclear structure is very important as it has been observed by comparing the two systems ${ }^{48} \mathrm{Ti}+{ }^{58} \mathrm{Fe}$ and ${ }^{58} \mathrm{Ni}+$ ${ }^{54} \mathrm{Fe}[11,12]$ (see Fig. 2). We notice that the two behaviours are remarkably different; indeed, the slope of ${ }^{48} \mathrm{Ti}$ $+{ }^{58} \mathrm{Fe}$ saturates below the barrier and remains much lower than $L_{C S}$. No maximum of the $S$ factor develops, so that, in other words, no fusion hindrance seems to show up in this case in the measured energy range. On the other hand a clear maximum of the $S$ factor can be observed for ${ }^{58} \mathrm{Ni}+{ }^{54} \mathrm{Fe}$. The different trends originate from the dissimilar low energy nuclear structure of the involved nuclei. Indeed, ${ }^{48} \mathrm{Ti}$ and ${ }^{58} \mathrm{Fe}$ are soft and have a low-lying quadrupole excitation lying at $800-900 \mathrm{keV}$ only. Instead, ${ }^{58} \mathrm{Ni}$ and ${ }^{54} \mathrm{Fe}$ have a closed shell (protons and neutrons, respectively) and are rather stiff.

\section{The two systems ${ }^{12} \mathbf{C}+{ }^{30} \mathrm{Si},{ }^{24} \mathrm{Mg}$ : sub-barrier trends of the excitation functions and of the $S$-factors}

A different situation might be found in medium-light and light systems where the $Q$-value for fusion may be positive. Indeed in such cases there is no energy threshold for fusion and consequently it is not necessary that the $S$ factor develops a maximum. Whether there is also an $S$ factor maximum at very low energies for such systems is an experimentally challenging question and various experiments have been recently performed in this sense (see e.g. Ref. [13]). However the existence of an $S$ factor maximum has not yet been clearly established. Recently a detailed study on the system ${ }^{12} \mathrm{C}+{ }^{30} \mathrm{Si}\left(Q_{f u s}=+14.1 \mathrm{MeV}\right)$ in a wide energy range showed an empirical evidence of the hindrance effect because the slope reaches and overcomes the $L_{C S}$ value and the $S$ factor appears to develop a maximum with decreasing energy. This is shown in Fig. 3 and is supported by CC calculations within the adiabatic model [14], using both the WS and the YPE potentials [15]. A damping of the coupling strengths improves the data fit at low energies and, overall, the YPE potential gives better results. The hindrance effect is weak but clearly observable from the comparison with the calculations.

The near-by system ${ }^{12} \mathrm{C}+{ }^{24} \mathrm{Mg}\left(Q_{\text {fus }}=+16.3 \mathrm{MeV}\right)$ is even closer to the cases of astrophysical interest. Preliminary LNL data extending much below the barrier are reported in Fig. 4 that combines the trend of both excitation function and of the $S$ factor. The $S$ factor develops a 

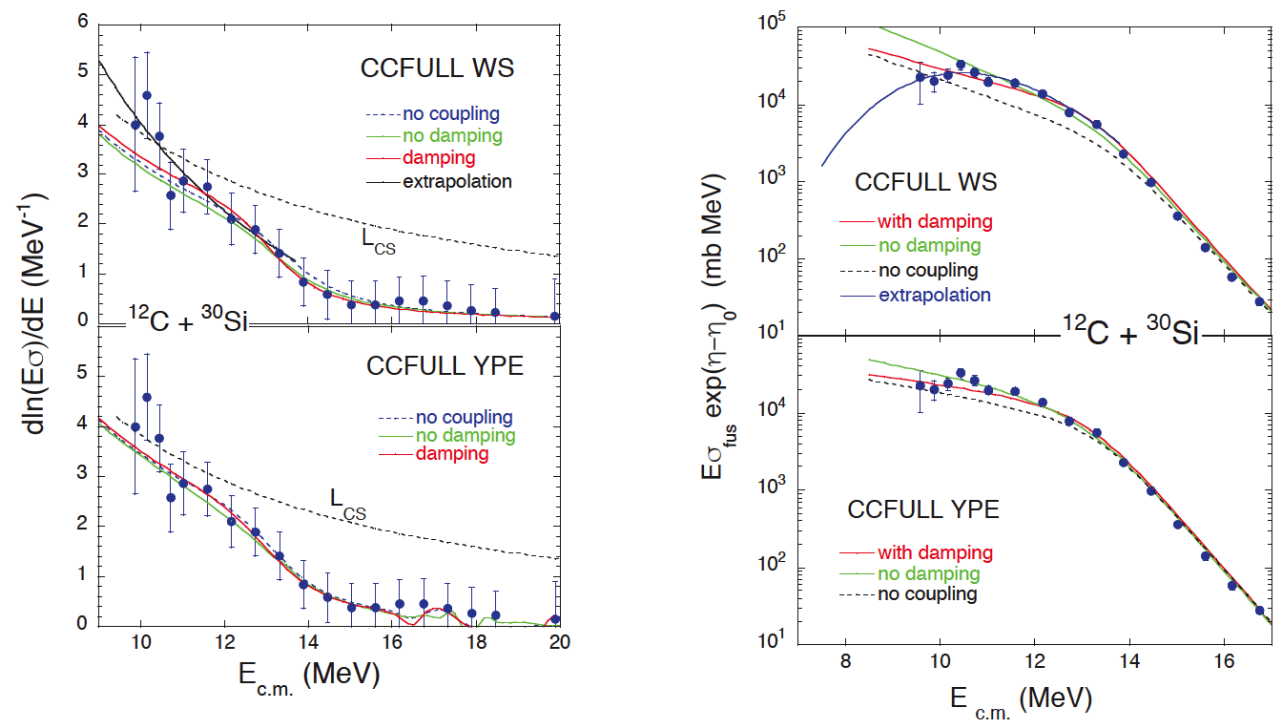

Figure 3. The logarithmic slope (left) crosses the $L_{C S}$ value at the lowest energies where the $S$ factor (right) appears to develop a maximum. In both cases a phenomenological extrapolation is shown, based on the systematics [16] (black and blue lines respectively).

clear maximum at about $9 \mathrm{MeV}$, indicating the presence of hindrance.

It is interesting to note that the cross section corresponding to the hindrance threshold is very high $\left(\sigma_{s}=1.6 \mathrm{mb}\right)$, probably the highest measured so far. This is reported in Fig. 5 together with several other medium-light systems. The high cross section at threshold has allowed to identify rather clearly the onset of hindrance, and it may also be possible to extend the measurements further down in energy.

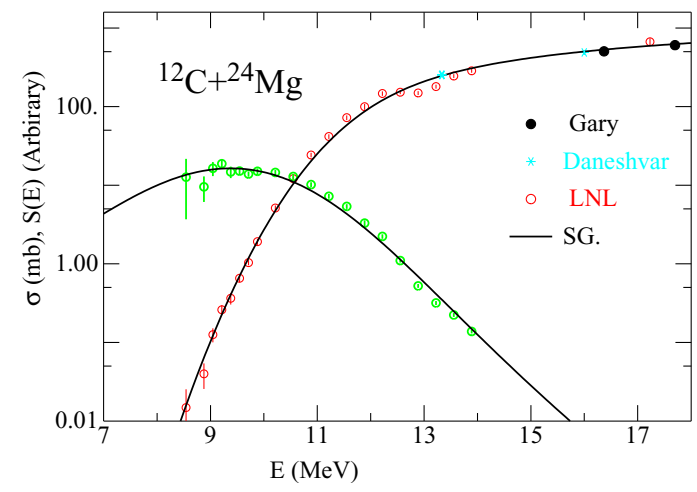

Figure 4. Excitation function and $S$ factor for ${ }^{12} \mathrm{C}+{ }^{24} \mathrm{Mg}$, previous data are also reported $[17,18]$. The curves are least-square fits of the excitation function.

\section{Towards light systems and possible consequences for astrophysics}

Concerning the two systems that I have just discussed in the previous Section, their energy threshold for hindrance $\left(E_{s}\right)$ nicely fits in the phenomenological systematics that

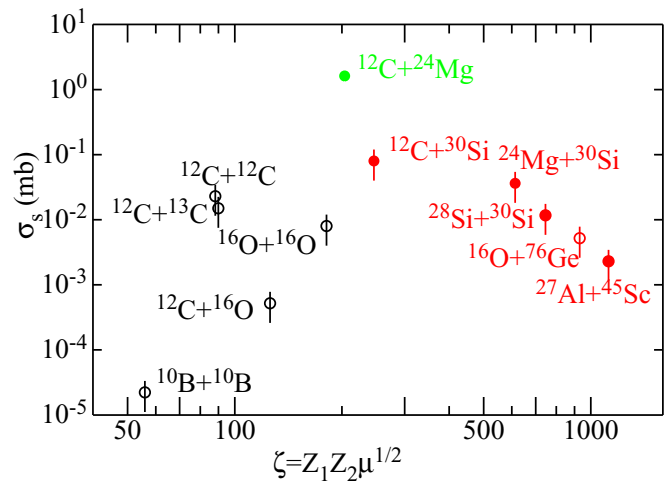

Figure 5. The threshold cross section vs. the system parameter $\zeta$ for ${ }^{12} \mathrm{C}+{ }^{24} \mathrm{Mg}$ and several other cases for comparison.

was developed by Jiang et al. a few years ago [16], and that is shown in Fig. 6. This gives us confidence that the extrapolation towards lighter systems as ${ }^{12} \mathrm{C}+{ }^{12} \mathrm{C}$ and ${ }^{16} \mathrm{O}$ $+{ }^{16} \mathrm{O}$ using that systematics, is reliable.

I want to stress another point concerning the difference of logarithmic derivatives between heavy and light systems, using the representation of Fig. 7. In each case the $Q$ value for fusion is indicated, as well as the system parameter $\zeta$. The dashed line is $L_{C S}(E)$, the red one is a simple extrapolation and the green lines are standard CC calculations. For lighter systems, $L(E)$ and $L_{C S}(E)$ are two nearly parallel or overlapping curves so the crossing point is rather undetermined and the $S$ factor maximum becomes broader and more difficult to be identified. For example, we notice that the slope of ${ }^{64} \mathrm{Ni}+{ }^{64} \mathrm{Ni}$ is very steep and crosses $L_{C S}$ with a large angle. Here the existence and the threshold of hindrance are quite well identified. On the contrary, in the light case of ${ }^{10} \mathrm{~B}+{ }^{10} \mathrm{~B}$ the 


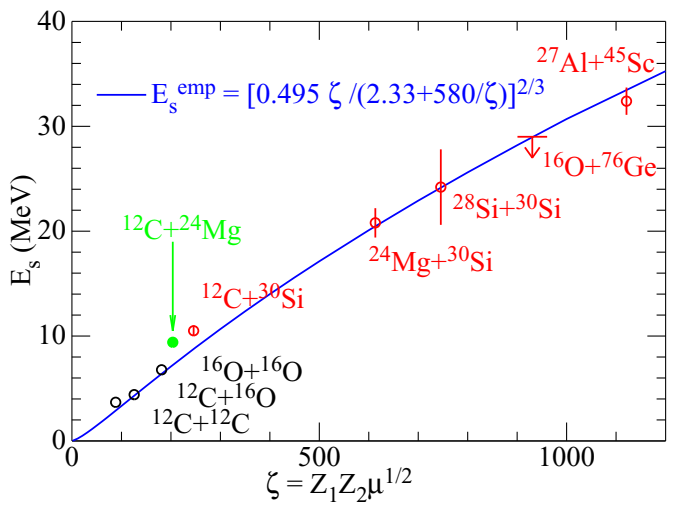

Figure 6. Systematics of $E_{s}$ in several light- and medium-light mass systems [16]. The locations for ${ }^{12} \mathrm{C}+{ }^{30} \mathrm{Si}$ and ${ }^{12} \mathrm{C}+{ }^{24} \mathrm{Mg}$ are very close to the astrophysically relevant cases, for which several data sets exist, but they are sometimes contradictory and the errors large. This results in large uncertainties in the expected values for their hindrance threshold. The corresponding points (open symbols) have therefore been obtained from extrapolations.

two curves $L(E)$ and $L_{C S}$ tend to overlap with each other at low energies.

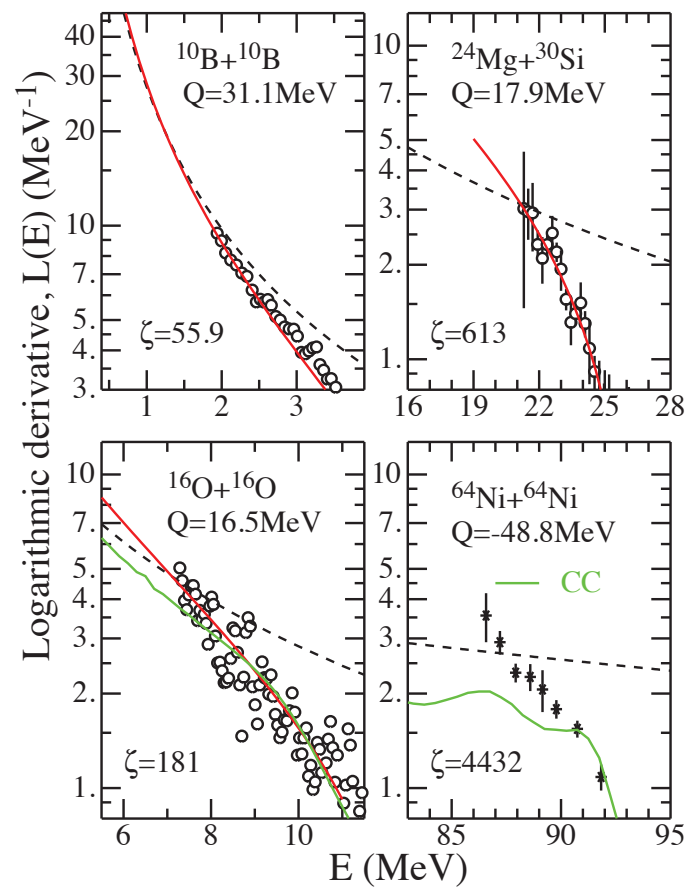

Figure 7. Logarithmic derivative for various heavy and light systems. In each case the Q-value for fusion is indicated, as well as the system parameter $\zeta=Z_{1} Z_{2} \mu^{1 / 2}$. The dashed line is $L_{C S}(\mathrm{E})$, the red one is a simple extrapolation and the green lines are standard CC calculations (courtesy of C.L. Jiang).

By introducing the quantity [9]

$$
R R=\frac{d L(E) / d E}{d L_{C S}(E) / d E}
$$

this trend is well represented (see Fig. 8, upper panel). RR is the ratio of the energy derivatives of the slopes $L(E)$ and $L_{C S}(\mathrm{E})$ at the crossing point. A sharp intersection point is observed for the heavier systems so that a well defined $S$ factor maximum develops. Instead, RR approaches unity for the lighter systems for which a less well defined maximum shows up. This does not imply that the fusion hindrance disappears in such cases, however, its existence may be more easily evidenced from comparing with standard CC calculations.

Shrivastava et al. [20] made recent studies of the way fusion hindrance shows up and evolves with increasing mass and charge of light projectiles like ${ }^{6,7} \mathrm{Li}$ and ${ }^{12} \mathrm{C}$ on the heavy target ${ }^{198} \mathrm{Pt}$. The case of ${ }^{16} \mathrm{O}+{ }^{208} \mathrm{~Pb}[21,22]$ was also re-analysed. They measured fusion cross sections down to very small values $(\approx 100 \mathrm{nb})$ using an off-line method by detecting coincidences between characteristic $\mathrm{X}$ - and $\gamma$-rays (see also [23]).

Fig. 8 (lower panel) shows that the hindrance effect is not observed for ${ }^{6,7} \mathrm{Li}$, but appears and becomes more evident when going to the heavier ${ }^{12} \mathrm{C}$ and ${ }^{16} \mathrm{O}$ projectiles. On the basis of this observed trend, it seems very important to study light and more symmetric systems where the existence of the hindrance phenomenon is still unclear and a matter of debate.

The very important case, from the astrophysical point of view, is the system ${ }^{12} \mathrm{C}+{ }^{12} \mathrm{C}$ for which several sets of experimental data already exist. For this case, in a recent experiment performed at Argonne [24] coincidences between $\alpha$ and $p$ particles, and $\gamma$-rays were detected. These data suggest that a maximum of the $S$ factor seems to develop around 3.5-4 MeV, in agreement with the phenomenological estimate of Ref. [16]. The S factor has been also recently deduced, using the indirect Trojan Horse Method, down to very low energies [25]. The many resonances observed in this experiment produce an overall increase of the $S$ factor below the energy range covered by direct measurements and no indication of hindrance appears.

\section{Summary and conclusions}

The phenomenon of hindrance in sub-barrier heavy-ion fusion was discovered about 15 years ago and it is presently established to be a general phenomenon. It is recognised in many cases by the trend of the logarithmic slope and of the $S$ factor, that generally develops a maximum vs. energy far below the barrier. In any case the comparison of the excitation function with standard CC calculations is a more quantitative evidence for the existence of the hindrance effect. For medium-heavy systems where the $Q$ value for fusion is negative, the existence of hindrance is necessary because the $S$ factor vanishes at $E=-Q$. The interest in these cases is related mainly to the threshold of the effect that may strongly depend on the nuclear structure of the colliding ions.

Light systems mostly have positive $Q$ value for fusion so that the $S$ factor does not necessarily show a maximum at some energy. Several experiments have been recently performed to clarify this point because the existence of 

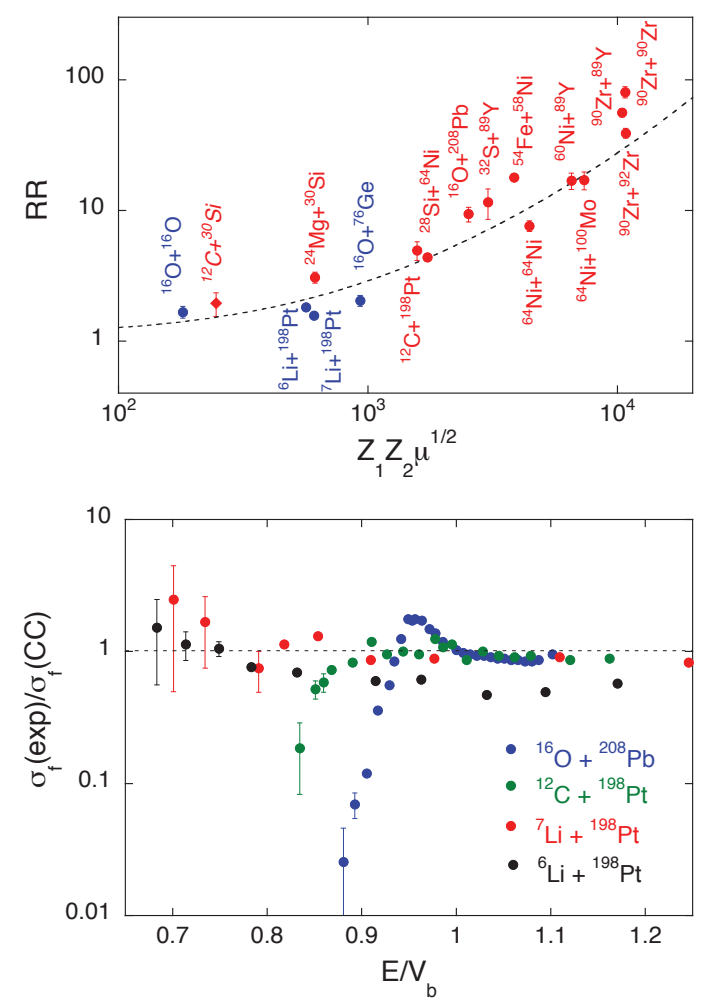

Figure 8. (upper panel) Ratio (RR) of energy derivatives of the slopes $L(E)$ and $L_{C S}(\mathrm{E})$ at their crossing points, vs. the system parameter $\zeta=Z_{1} Z_{2} \mu^{1 / 2}$. The red dots are extracted from experimental data while the blue ones are extrapolated from data at higher energies. The red diamond is the value for ${ }^{12} \mathrm{C}+{ }^{30} \mathrm{Si}$ [15]. The dashed line is the curve resulting from the systematics discussed in Ref. [9]. (lower panel) Measured vs. calculated fusion cross sections as a function of the energy relative to the Coulomb barrier for ${ }^{6,7} \mathrm{Li}+{ }^{198} \mathrm{Pt}[19,20],{ }^{12} \mathrm{C}+{ }^{198} \mathrm{Pt} \mathrm{[20]} \mathrm{and}{ }^{16} \mathrm{O}+$ ${ }^{208} \mathrm{~Pb}[21,22]$ systems. The calculated values $\left(\sigma_{f}(\mathrm{CC})\right)$ correspond to CCFULL calculations using a standard WS potential. Figure redrawn from Ref. [20].

hindrance in such light cases may have relevant consequences in astrophysics. Indeed the phase of carbon burning in massive stars might be substantially influenced, with a reduction of predicted astrophysical reaction rates.

So far hindrance has been possibly observed in various medium-light systems, independent of the sign of the $Q$-value. The hindrance effect is small but it has been clearly recognised in the two recent experiments on ${ }^{12} \mathrm{C}$ $+{ }^{30} \mathrm{Si},{ }^{24} \mathrm{Mg}$ that are very close to the astrophysically relevant systems, and its threshold nicely follows a systematics proposed a few years ago. The consequences for the dynamics of stellar evolution have to be clarified by further experimental and theoretical work.

\section{Acknowledgments}

I am grateful to all colleagues who have collaborated in the experiments presented in this talk for their invaluable help. Special thanks are addressed to XTU Tandem staff for their professional work. I also wish to acknowledge many other experimental and theoretical colleagues for their precious contributions to several aspects of the work presented here.

\section{References}

[1] N. Rowley, G.R. Satchler and P.H. Stelson, Phys. Lett. B 254, 25 (1991).

[2] W. Reisdorf, F.P. Hessberger, K.D. Hildebrand, S. Hofmann, G. Münzenberg, K.-H. Schmidt, J.H.R. Schneider, W.F.W. Schneider, K,Sümmerer, G. Wirth, J.V. Kratz and K. Schlitt, Nucl. Phys. A 438, 212 (1985).

[3] A. M. Stefanini, D. Ackermann, L. Corradi, D. R. Napoli, C. Petrache, P. Spolaore, P. Bednarczyk, H. Q. Zhang, S. Beghini, G. Montagnoli, L. Mueller, F. Scarlassara, G. F. Segato, F. Soramel and N. Rowley, Phys. Rev. Lett. 74, 864 (1995).

[4] A. M. Stefanini, B. R. Behera, S. Beghini, L. Corradi, E. Fioretto, A. Gadea, G. Montagnoli, N. Rowley, F. Scarlassara, S. Szilner, and M. Trotta, Phys. Rev. C 76, 014610 (2007).

[5] L.R. Gasques, E.F. Brown, A. Chieffi, C.L. Jiang, M. Limongi, C. Rolfs, M. Wiescher, and D.G. Yakovlev, Phys. Rev. C 76, 035802 (2007).

[6] B.B. Back, H. Esbensen, C.L. Jiang, and K.E. Rehm, Rev. Mod. Phys. 86, 317 (2014).

[7] C. L. Jiang, H. Esbensen, B. B. Back, R. V. F. Janssens, and K. E. Rehm, Phys. Rev. C 69, 014604 (2004).

[8] C. L. Jiang, B. B. Back, H. Esbensen, R. V. F. Janssens, and K. E. Rehm, Phys. Rev. C 73, 014613 (2006).

[9] C. L. Jiang, B. B. Back, R. V. F. Janssens, and K. E. Rehm, Phys. Rev. C 75, 057604 (2007).

[10] C.L.Jiang, K. E. Rehm, R.V. F. Janssens, H. Esbensen, I. Ahmad, B. B. Back, P. Collon, C. N. Davids, J. P. Greene, D. J. Henderson, G. Mukherjee, R. C. Pardo, M. Paul, T.O. Pennington, D. Seweryniak, S. Sinha, and Z. Zhou, Phys. Rev. Lett. 93, 012701 (2004).

[11] A.M. Stefanini, G. Montagnoli, L. Corradi, S. Courtin, E. Fioretto, A. Goasduff, F. Haas, P. Mason, R. Silvestri, Pushpendra P. Singh, F. Scarlassara, and S. Szilner, Phys. Rev. C 82, 014614 (2010).

[12] A.M. Stefanini, G. Montagnoli, L. Corradi, S. Courtin, D. Bourgin, E. Fioretto, A. Goasduff, J. Grebosz, F. Haas, M. Mazzocco,T. Mijatović, D. Montanari, M. Pagliaroli, C. Parascandolo, F. Scarlassara, E. Strano, S. Szilner, N. Toniolo, and D. Torresi, Phys. Rev. C 92, 064607 (2015).

[13] G. Montagnoli and A.M. Stefanini, Eur. Phys. J. A 53, 169 (2017).

[14] T. Ichikawa, Phys. Rev. C 92, 064604 (2015).

[15] G. Montagnoli, A. M. Stefanini, C. L. Jiang, K. Hagino, F. Galtarossa, G. Colucci, S. Bottoni, C. Broggini, A. Caciolli, P. Čolović, L. Corradi, S. Courtin, R. Depalo, E. Fioretto, G. Fruet, A. Gal, A. Goasduff, M. Heine, S. P. Hu, M. Kaur, T. Mijatović, M. Mazzocco, D. Montanari, F. Scarlassara, E. Strano, S. Szilner, and G. X. Zhang, Phys. Rev. C 97, 024610 (2018). 
[16] C. L. Jiang, K. E. Rehm, B. B. Back and R. V. F. Janssens, Phys. Rev. C 79, 044601 (2009).

[17] S.Gary and C. Volant, Phys. Rev. C 25, 1877 (1982).

[18] K. Daneshvar, D. G. Kovar, S. J. Krieger, and K. T. R. Davies, Phys. Rev. C 25, 1342 (1982).

[19] A. Shrivastava, A. Navin, A. Lemasson, K. Ramachandran, V. Nanal, M. Rejmund, K. Hagino, T. Ichikawa, S. Bhattacharyya, A. Chatterjee, S. Kailas, K. Mahata, V. V. Parkar, R. G. Pillay, and P. C. Rout, Phys. Rev. Lett. 103, 232702 (2009).

[20] A. Shrivastava, K. Mahata, S.K. Pandit, V. Nanal, T. Ichikawa, K. Hagino, A. Navin, C.S. Palshetkar, V.V. Parkar, K. Ramachandran, P.C. Rout, Abhinav Kumar, A. Chatterjee, and S. Kailas, Phys. Lett. B 755, 332 (2016).

[21] M. Dasgupta, D. J. Hinde, A. Diaz-Torres, B. Bouriquet, Catherine I. Low, G. J. Milburn, and J. O. Newton, Phys. Rev. Lett. 99, 192701 (2007).

[22] C.R. Morton, A.C. Berriman, M. Dasgupta, D.J. Hinde, J.O. Newton, K. Hagino and I.J. Thompson, Phys. Rev. C 60, 044608 (1999).
[23] A. Lemasson, A. Shrivastava, A. Navin, M. Rejmund, V. Nanal, S. Bhattacharyya, A. Chatterjee, S. Kailas, K. Mahata, V.V. Parkar, R.G. Pillay, K. Ramachandran, and P.C. Rout, Nucl. Instr. Meth. Phys. Res. A 598, 445 (2009).

[24] C. L. Jiang, D. Santiago-Gonzalez, S. AlmarazCalderon, K. E. Rehm, B. B. Back, K. Auranen, M. L. Avila, A. D. Ayangeakaa, S. Bottoni, M. P. Carpenter, C. Dickerson, B. DiGiovine, J. P. Greene, C. R. Hoffman, R. V. F. Janssens, B. P. Kay, S. A. Kuvin, T. Lauritsen, R. C. Pardo, J. Sethi, D. Seweryniak, R. Talwar, C. Ugalde, S. Zhu, D. Bourgin, S. Courtin, F. Haas, M. Heine, G. Fruet, D. Montanari, D. G. Jenkins, L. Morris, A. Lefebvre-Schuhl, M. Alcorta, X. Fang, X. D. Tang, B. Bucher, C. M. Deibel, and S. T. Marley Phys. Rev. C 97, 012801(R) (2018).

[25] A. Tumino, C. Spitaleri, M. La Cognata, S. Cherubini, G. L. Guardo, M. Gulino, S. Hayakawa, I. Indelicato, L. Lamia, H. Petrascu, R. G. Pizzone, S. M. R. Puglia, G. G. Rapisarda, S. Romano, M. L. Sergi, R. Spartá and L. Trache, Nature 557, 687 (2018). 